\title{
EVALUATION OF LUMINOSITY REDUCTION IN THE ILC HEAD-ON SCHEME FROM PARASITIC COLLISIONS
}

\author{
J. Brossard", P. Bambade, T. Derrien, LAL, Université Paris-Sud, IN2P3-CNRS, Orsay, B.P. 34, \\ 91898, Orsay cedex, France \\ O. Napoly, J. Payet, CEA, Gif-sur-Yvette, France, \\ M.C. Alabau, IFIC, Valencia, Spain D. Angal-Kalinin, STFC/DL/ASTeC, Daresbury, Warrington, \\ Cheshire, UK.
}

\begin{abstract}
An interaction region with head-on collisions is being developed for the ILC as an alternative to the base line 14 mrad crossing angle design, motivated by simpler beam manipulations upstream of the interaction point (IP) and a more favourable configuration for the detector and physics analysis. The design of the post-collision beam line in this scheme involves however a number of technological challenges, one of which is the strength requirement for the electrostatic separators (ES) placed immediately after the final doublet to extract the spent beam. In this paper, we examine in detail the main mechanism behind this requirement, the multi-beam kink instability, which results from the long-range beam-beam forces at the parasitic crossings after the bunches have been extracted. Our analysis uses realistic bunch distributions, the Guinea-Pig program to treat beam-beam effects at the interaction point and the DIMAD program to track the disrupted beam distributions in the postcollision beam line.
\end{abstract}

\section{ELECTROSTATIC SEPARATOR REQUIREMENT}

In the current head-on scheme, the first element used to deviate the trajectory of the bunches after collision is the electrostatic separator. The design of this device should satisfy 2 main requirements (Figure 1). The first one is imposed by the influence of the electromagnetic perturbation at the first parasitic crossing (at this location, the horizontal separation between the outgoing and the incoming bunches is not large enough to design 2 separate vacuum chamber). The second one is the horizontal offaxis position of the disrupted bunch inside the third quadrupole of the extraction line. This position determines - with the horizontal bunch size - a lower limit for the inner aperture of the quadrupole, and the dipole horizontal kick induced on the disrupted bunch.

The main purpose of this study is to defined - for a given amplitude vertical jitter at the IP - the minimal distance at the first parasitic crossing inducing an acceptable luminosity reduction $\left(\mathrm{L} / \mathrm{L}_{0} \geq 95 \%\right)$. Excepted for the "Low Power" parameter set the time space between 2 consecutives bunches is greater or equal than 369.2 ns [1]. Thus, the first parasitic crossing location occurs - for nominal parameter (NP) set - at $55.34 \mathrm{~m}$ from the IP. In the current analysis, the parasitic location is assumed to be at $56.03 \mathrm{~m}$.

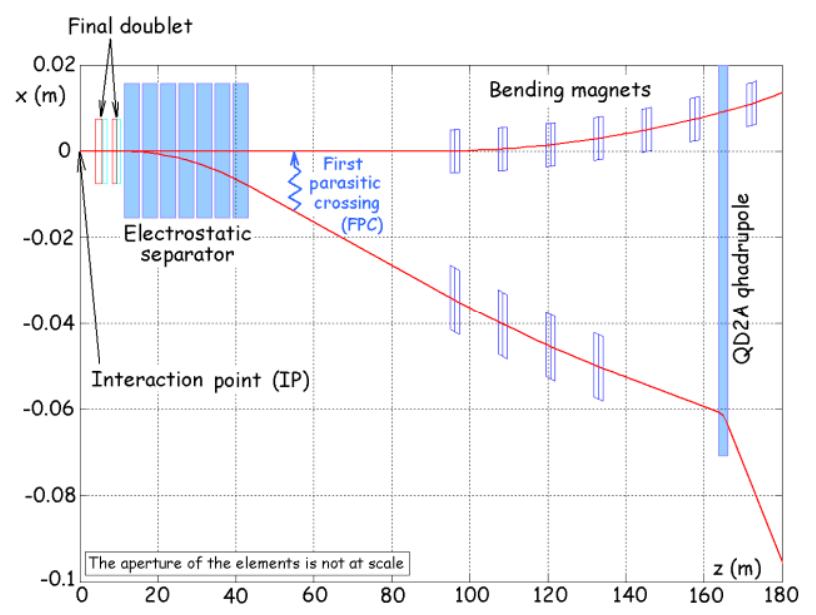

Figure 1: Top view of the head-on extraction line.

\section{MULTI-BUNCH KINK INSTABILITY}

\section{Previous Analysis}

In a previous multi-bunch kink instability study performed by O. Napoly, J. Payet \& al. [2], the beambeam effect at the IP and the incoherent beam-beam effect at the first parasitic crossing point were not studied. In this study, the disrupted bunch was approximated using the single nominal macro-particle moving under linear optics condition, and no beam-beam simulation was made. The collision effect was approximated by using the linear regime of the vertical beam-beam deflection curve [3]. The results shows that for a $0.5 \sigma_{\mathrm{y}}$ vertical jitter at the IP, and a $10.97 \mathrm{~mm}$ horizontal distance at the first parasitic crossing, the luminosity reduction induced by Kink effect is lower than $5 \%$ (for NP at $500 \mathrm{GeV}$ in center of mass).

The present analysis goal is to check the possible modification of this limit value based on a more accurate modelling ( $2^{\text {nd }}$ order tracking of "real" disrupted distribution in the extraction line and complete beambeam simulation).

\footnotetext{
\#brossard@1al.in2p3.fr
} 


\section{BEAM-BEAM EFFECT}

In the current analysis, we performed the beam-beam effect (using 50000 macro-particles) analysis using the dedicated "Guinea-Pig" software [4]. The beam-beam effect modelling induced the creation of a long low energy tail in the energy macro-particles distribution (down to $-50 \%$ of nominal energy, for nominal parameter) and the increasing the horizontal angular divergence. Moreover, the vertical deflection after collision is a non linear function of the vertical offset (Figure 2).

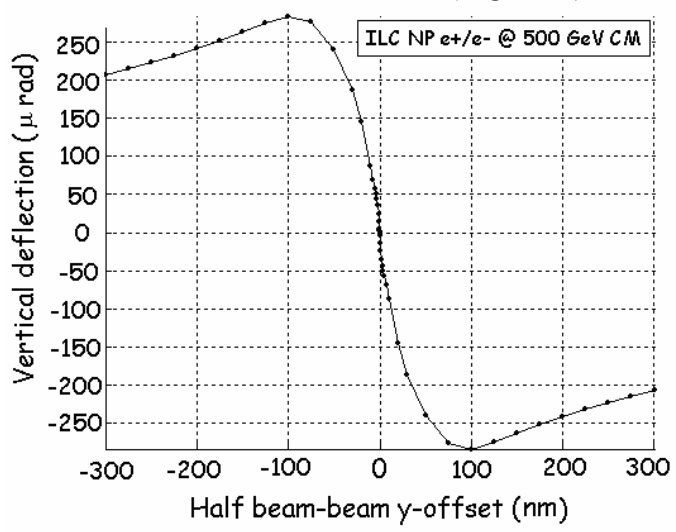

Figure 2: Vertical deflection angle versus vertical half beam-beam offset, for e+e- collision at the ILC with nominal parameters at $500 \mathrm{GeV}$ in the center-of-mass [3].

The current analysis, performed for different standard deviation of the vertical jitter $\left(\sigma_{\mathrm{jy}}=0.1,0.5,1,3\right.$ and $\left.5 \mathrm{~nm}\right)$ and different horizontal distance between incoming and outgoing nominal particle at the FPC $(\delta x=3$ to $10 \mathrm{~mm}$ with $1 \mathrm{~mm}$ step) induced a large range of vertical offset value. Thus a preliminary task was to automate Guinea Pig. This was done using a shell script MATLAB scripts which varied the desired parameters using the rules defined in Table 1 . Within such parameterisation, the precision of the beam-beam simulation is acceptable (the "miss1" and "miss2" Guinea-Pig parameters are most of the time lower than $20 \%$, excepted for $\delta \mathrm{x}=3 \mathrm{~mm}$ where $50 \%$ value is reached).

Table 1: Guinea-Pig “y-grid” Scaling

\begin{tabular}{|l|c|c|}
\hline & n_y & cut_y \\
\hline Abs(offset-y @ IP) $\leq 0.1 \mathrm{~nm}$ & 128 & 6 \\
\hline $0.1 \mathrm{~nm}<$ Abs(offset-y @ IP) $\leq 10 \mathrm{~nm}$ & 256 & 20 \\
\hline $10 \mathrm{~nm}<$ Abs(offset-y @ IP) & 512 & 24 \\
\hline
\end{tabular}

\section{FIRST PARASITIC PERTURBATION}

The perturbation induced by the outgoing bunch on the next incoming bunch at the FPC is computed assuming that all individual macro-particles represents a z-axis cylindrical gaussian distribution, with a radial $\sigma$ small compared to the distance of the nominal injection line particle. Under this assumption, the perturbation on the incoming nominal macro-particle could be approximated by an angular vertical kick $\alpha_{\mathrm{y}}$ given by :

$$
\alpha_{y}=-\frac{2 R_{e}}{\gamma} \frac{N_{\text {particles }}}{N_{\text {macroparticles }}} \sum_{i=1}^{N_{\text {macroparticles }}} \frac{y_{i}}{\left(x_{i}-\delta x\right)^{2}+y_{i}^{2}}
$$

Where $\mathrm{R}_{\mathrm{e}}=2.8179 \mathrm{e}-15 \mathrm{~m}$ is the classical electron radius, $\gamma$ is the Lorentz factor, $\mathrm{N}_{\text {particles }}$ is the number of particles/bunch, and $\mathrm{N}_{\text {macroparticles }}$ is the number of macroparticles used in the extraction line tracking ( $\sim 50000$ in this study). The disrupted incoming nominal particle is then tracked to the IP through the injection line. At the IP, a vertical offset jitter is added to the vertical position of the nominal particle, and the total vertical offset (assumed to be the half beam-beam offset) is used in Guinea-Pig to perform a new beam-beam simulation.

\section{RESULTS}

For each $\sigma_{\mathrm{jy}}$ and $\delta \mathrm{x}$ values, the loop simulation (Figure 3 ) has been used to analyse 20 consecutives collisions. The result shows that this number is large enough to reach a stabilised regime for the luminosity. In order to estimate the luminosity reduction induced by the FPC perturbation, the luminosity has been computed for perturbed and unperturbed bunches. The bunch to bunch random guineapig macro-particles generation induces a numerical dispersion on the luminosity lower than $1.2 \%$. Thus, all variation in relative luminosity lower than this value is not relevant. When the first parasitic crossing impact on luminosity is important, a stabilised regime is obtained after 5 collisions. Thus, in order to quantitatively estimate the FPC effect, the mean luminosity (for a given $\sigma_{\mathrm{jy}}$ and $\delta \mathrm{x}$ values) is computed by averaging the last 15 collisions.

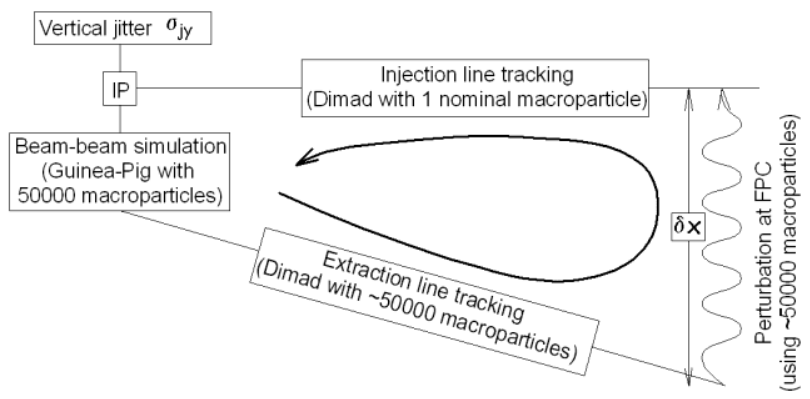

Figure 3: Scheme of the simulation loop.

Figure 4 shows the ratio between the mean luminosity induced by the vertical jitter at the IP and the mean luminosity induced by the jitter plus the perturbation at the FPC. This plot shows that for beam separation greater than $6.5 \mathrm{~mm}$, the FPC perturbation effect on luminosity is insignificant. Below $5 \mathrm{~mm}$, the relative luminosity decrease and reach a $10 \%$ at $3 \mathrm{~mm}$.

A previous analysis, using single macro-particle in the extraction line, and curve of Figure 2 for the beam-beam effect modelling has been realized. The result of this analysis are shown on Figure 5. The region of insignificant FPC effect on luminosity is identical as the 
"guinea-Pig + DIMAD 50000 macro-particles" analysis. Only the slope of the lower part of the curve $(\delta \mathrm{x}<6 \mathrm{~mm})$ is modified.

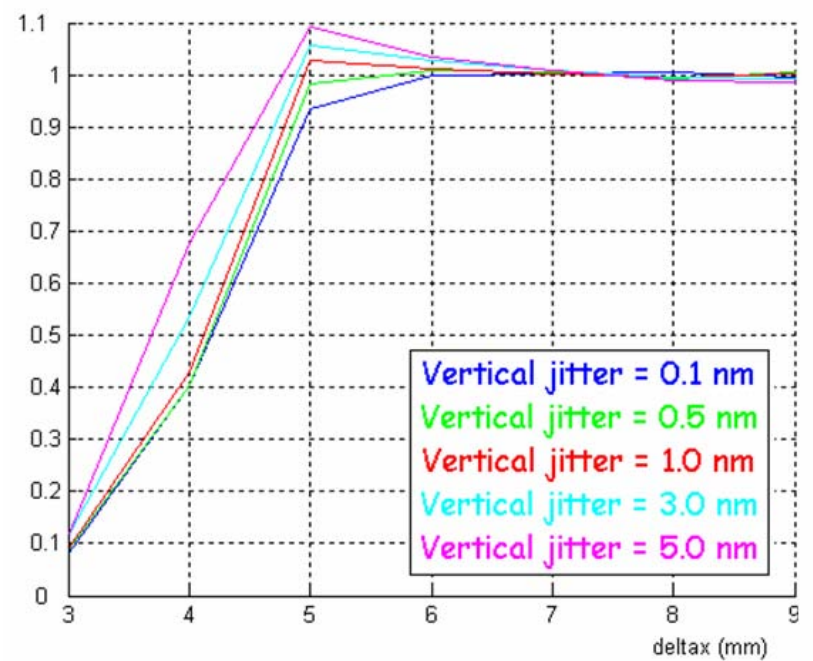

Figure 4: Relative luminosity ( $\mathrm{L} 0 / \mathrm{L}_{\text {with }}$ KINK), for a "Guinea-Pig + DIMAD 50000 macro-particles" modelling versus distance between bunches at the FPC (Nominal parameter at $500 \mathrm{GeV}$ center of mass).

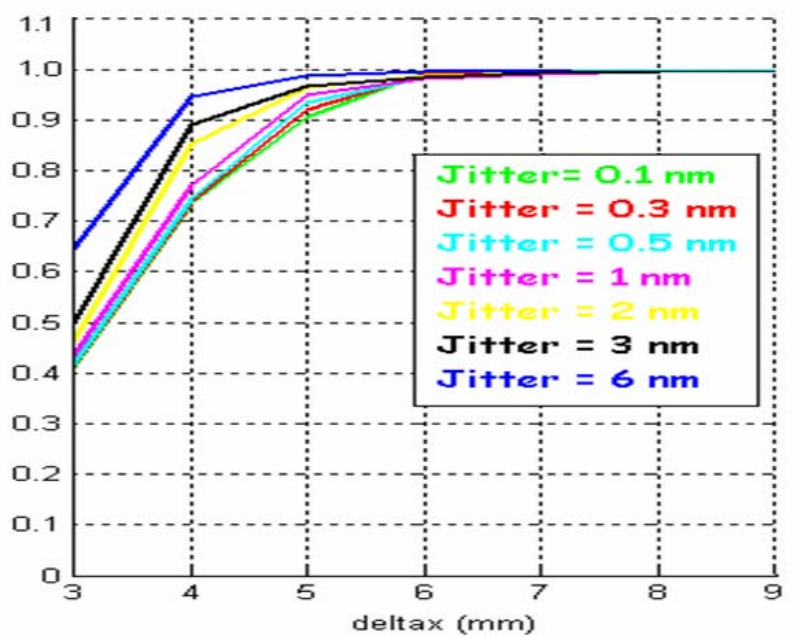

Figure 5: Relative luminosity (L0/ $\mathrm{L}_{\text {with KINK }}$ ), for a "one macro-particle" modelling versus distance between bunches at the FPC (Nominal parameter at $500 \mathrm{GeV}$ center of mass).

\section{CONCLUSIONS}

A long-range electromagnetic perturbation analysis has been performed at the first parasitic crossing in order to determine the minimal horizontal separation between incoming and outgoing bunches for nominal parameter set of head-on scheme at $500 \mathrm{GeV}$ center of mass. This horizontal threshold is a key parameter to design the strength of the electrostatic separator. In the current analysis, the bunch collision is performed using GuineaPig software. The extraction and injection line between IP and first parasitic crossing has been realized using $2^{\text {nd }}$ order DIMAD tracking code.

This simulation, based on 20 consecutives collisions of 50000 macro-particles shows that the minimal distance at the first parasitic crossing should be greater than $6 \mathrm{~mm}$. The results are largely independent of the magnitude of the vertical jitter at the IP. In the future, this analysis will be applied to study two potentially more dangerous cases: operation with High Luminosity or Low Power beam parameters and at lower center-of-mass energy.

\section{ACKNOWLEDGMENT}

This work is supported by the Commission of the European Communities under the Framework Programme "Structuring the European Research Area", contract number RIDS-011899.

\section{REFERENCES}

[1] http://media.linearcollider.org/rdr_draft_v1.pdf

[2] O. Napoly, J.Payet, C. Rippon, Evaluation of the Multi-Bunch Kink Instability in the ILC Head-on Collisions, EUROTeV-Report-2006-018, April 2006, http://www-ist.cea.fr/publicea/exldoc/200600003732.pdf .

[3] M. Alabau Pons, Optimization of the e-e- option for the ILC, EPAC'06, Edimburgh, June 2006, p. 685687 ,

http://accelconf.web.cern.ch/AccelConf/e06/PAPER S/MOPLS061.PDF

[4] D. Schulte, Ph. D. Thesis, University of Hamburg 1996,

TESLA-97-08, http://flash.desy.de/sites/site_vuvfel/content/e403/e16 44/e1314/e1316/infoboxContent1932/tesla199708.pdf 